\title{
Composition des 3 types de foie gras : oie, canard mulard et canard de Barbarie
}

\author{
MR Salichon 1, G Guy 2, D Rousselot 2, JC Blum ${ }^{1}$ \\ 1 INRA, station de recherches avicoles, 37380 Nouzilly; \\ 2 Station expérimentale des palmipèdes à foie gras, Artiguères, 40280 Benquet, France
}

(Reçu le 2 juin 1993; accepté le 24 novembre 1993)

\begin{abstract}
Résumé - Nous avons comparé les 3 types de foie gras commercialisés en France : les foies gras d'oie, de canard mulard et de canard de Barbarie. Ces foies gras ont été obtenus à partir d'animaux élevés en même temps dans des conditions semblables et gavés à l'âge de 12 (canards) ou 13 sem (oies des Landes) pendant $13 \mathrm{j}$ avec le même maïs : $11,7 \mathrm{~kg} / \mathrm{oie}$; $10,7 \mathrm{~kg} / \mathrm{canard}$ mulard et $8,1 \mathrm{~kg} /$ canard de Barbarie. Le poids des foies gras retenus pour cette étude ( 9 foies par type de palmipèdes, tous de sexe mâle) est en moyenne de 768,677 et $553 \mathrm{~g}$ respectivement dans le cas des oies, des canards mulards et des canards de Barbarie. Les teneurs du foie en lipides sont significativement plus élevées chez les canards de Barbarie $(62,6 \%)$ et mulards $(60,5 \%)$ comparés à l'oie $(54,6 \%)$, la proportion des autres constituants (eau, protéines et cendres) varie en sens inverse. Les triglycérides constituent la plus grande partie des lipides du foie gras : $95,7 \%$ et $94,4 \%$ chez les canards de Barbarie et mulard, contre $92,2 \%$ chez l'oie. $\dot{A}$ l'inverse, les teneurs en phospholipides et cholestérol sont plus élevées chez l'oie : respectivement 3,5 et $3,2 \%$ contre 1,5 et $1,7 \%$ chez le canard de Barbarie $(P<0,001)$. Les pourcentages d'acides gras saturés sont toujours plus élevés dans le foie que dans le tissu adipeux, les teneurs en acide linoléique étant au contraire considérablement réduites : $1,2 \%$ des acides gras du foie chez les canards, $0,8 \%$ chez l'oie, contre 8 à $11 \%$ dans les tissus adipeux. La perte de graisse après chauffage du foie à $105^{\circ} \mathrm{C}$ pendant 50 min est plus faible pour l'oie (14\%) comparée au canard mulard $(41 \%)$ et surtout au canard de Barbarie $(56 \%)$. Ces variations importantes de la qualité technologique pourraient être liées aux modifications de la composition et, particulièrement, à l'équilibre entre la proportion de graisse et la part des autres constituants (protéines, phospholipides, etc).
\end{abstract}

canard / composition / foie gras / qualité / oie

Summary - Composition of the 3 types of foie gras: goose, mule duck and muscovy duck foie gras. We compared the 3 types of foie gras available in France: foie gras from Landes geese, mule ducks (muscovy $x$ common duck) and muscovy ducks. These foie gras were obtained by similar preparations from the various birds, using only males reared in the same place and at the same time and finally over-fed at age 12 weeks (ducks) or 13 weeks (geese) for $13 d$ with the same corn: $11.7 \mathrm{~kg} / \mathrm{goose}, 10.7 \mathrm{~kg} / \mathrm{mule}$ duck, $8.1 \mathrm{~kg} / \mathrm{muscovy}$ duck. Nine livers per type of bird were used in this study. On average, fatty livers weighed 768,677 and $553 \mathrm{~g}$ respectively for geese, 
mule and muscovy ducks. The lipids contents were significantly $(P<0.001)$ higher in duck foie gras (62.6\% in muscovy and $60.5 \%$ in mule ducks vs $54.6 \%$ in geese), while, as reported in table 11 , water, protein and ash contents were lower. Triglycerides were the major component of lipids: $95.7 \%$ in muscovy and $94.4 \%$ in mule ducks vs $92.2 \%$ in geese (significant difference, $\mathrm{P}<0.001$ ). Phospholipids and cholesterol were naturally changed in the opposite direction being higher in geese: respectively 3.2 and $3.5 \%$ vs 1.5 and $1.7 \%$ in muscovy ducks. Fatty-acid analysis was carried out both in fatty livers and in adipose tissues. The percentages of saturated fatty acids were always found to be higher in livers than in adipose tissues, while those of linoleic acid were considerably reduced in livers: $1.2 \%$ in ducks, $0.8 \%$ in geese vs 8 to $11 \%$ in adipose tissues. The technological quality of foie gras was estimated by fat release after heating at $105^{\circ} \mathrm{C}$ for $50 \mathrm{~min}$. On average, the livers of muscovy ducks, hybrid ducks and geese lost respectively 56, 41 and $14 \%$ of their weight. Figure 2 shows that individual values depended on the liver weight only in the case of geese and hybrid ducks; in muscovy ducks every liver released the same maximum fat percentage (very poor quality) whatever its weight. These important technological quality variations could be related to liver composition and more precisely to the balance between fat and other components (phospholipids or proteins).

ducks / hepatic steatosis / goose / liver / over-feeding / quality

\section{INTRODUCTION}

La consommation française de foie gras de canard continue d'augmenter tandis que celle du foie gras d'oie semble se stabiliser.

Produit festif par excellence, le foie gras est réputé pour ses qualités gustatives et il demeure très apprécié malgré sa richesse en graisse; cependant la composition de ses lipides a été peu étudiée.

Parmi les 3 types de foies existant sur le marché, c'est celui de l'oie dont la composition est la mieux connue (Leclercq et al, 1969 ; Blum et Leclercq, 1973 ; Nir et Nitsan, 1976 ; Blum et al, 1990 ; Hermier et al, 1991) bien que la répartition de ses acides gras (Leclercq et al, 1968) n'ait été déterminée que sur quelques petits foies. Le foie de canard de Barbarie a été étudié à I'ENSA de Toulouse (Babilé, 1989 ; Rémignon, 1990 ; Baudonnet-Lenfant et al, 1991). Paradoxalement c'est le foie des canards mulards, de loin le plus produit et consommé en France, qui a été le moins étudié ; Auvergne (1992) apporte cependant des données chiffrées sur ses principaux constituants. Toutes ces études ont fait appel à des méthodes différentes, qu'il s'agisse des méthodes analytiques, de l'éle- vage, ou de la durée et de l'intensité du gavage. Nous avons voulu réaliser un essai simultané réunissant les différents palmipèdes producteurs de foie gras. Élevés, traités, préparés et gavés de la même façon (Guy et al, 1994), presque au même âge et avec le même maïs, les canards et les oies devraient fournir des produits comparables. Les mêmes méthodes d'analyse permettent d'éliminer l'hétérogénéité liée à l'usage de techniques différentes. Aussi, nous pensons que les données apportées ici permettent pour la première fois de reconnaître les analogies et les différences existant entre les 3 types de foie gras.

\section{MATÉRIEL ET MÉTHODES}

\section{Animaux et régimes}

Les techniques d'élevage utilisées sont rapportées en détail dans la communication de Guy et al (1993). Rappelons que les canards, tous mâles, comprennent 40 canards de Barbarie et 40 canards mulards qui sont mis en gavage à 12 sem à raison de 2 repas par jour. Les 40 oies sont mises en gavage à 13 sem à raison de 3 repas par jour avec repasse. La durée du gavage est de 13 j pour tous les animaux. Les quantités 
de maïs roux ingérées pendant la durée du gavage ont été respectivement de 11,$7 ; 10,7$ et $8,1 \mathrm{~kg}$ en moyenne par oie, canard mulard et canard de Barbarie.

\section{Étude analytique}

Les analyses portent sur 27 foies ( 9 d'oie, 9 de mulard, 9 de Barbarie) provenant tous d'animaux mâles choisis pour représenter au mieux (poids moyens des animaux et des foies \pm écarts types) chaque population. Les foies sont prélevés immédiatement après l'abattage (éviscération à chaud) et placés sur lit de glace; on prend $20 \mathrm{~g}$ dans le grand lobe de chaque foie aux fins d'analyse effectuée individuellement sur chaque foie. Six $\mathrm{g}$ environ de tissu adipeux abdominal sont également prélevés sur chaque animal. Après broyage et homogénéisation, les prélèvements de tissu adipeux sont réunis pour former 3 pools, représentant chacun 1 type de palmipède (oie, canard mulard ou canard de Barbarie).

Sur chaque échantillon de foie, on détermine : la teneur en protéines brutes par la méthode Kjeldahl adaptée au laboratoire (Kjeldahlterm et Vapodest Gerhart - titrator Mettler) ; la teneur en eau par séchage à l'étuve à $103^{\circ} \mathrm{C}$ pendant $8 \mathrm{~h}$; la matière minérale, mesurée par calcination à $550^{\circ} \mathrm{C}$ pendant $12 \mathrm{~h}$; les lipides totaux après extraction au chloroforme/méthanol (Folch et al, 1957) ; un aliquot de la fraction lipidique est utilisé pour la détermination des différentes classes de lipides par chromatographie en couche mince sur baguette (iatroscan) et les acides gras par chromatographie en phase gazeuse sur colonne capillaire après méthylation dans un mélange méthanol/acide sulfurique concentré (95-5) v/v.
Les 3 pools de tissus adipeux sont soumis à une analyse restreinte : extraction des lipides suivie par le dosage des acides gras en utilisant les mêmes techniques que celles mentionnées pour les foies.

\section{Méthodes statistiques}

Les 3 types de palmipèdes ont été comparés par une analyse de variance à un facteur, la signification des différences entre moyennes étant estimée par le test de Fisher.

\section{RÉSULTATS}

\section{Les animaux}

Le tableau I montre le poids des animaux retenus pour cette étude. Bien que les sujets les plus petits et les plus gros, à plus d'un écart type de la moyenne, aient été écartés, les poids vifs moyens sont proches de ceux indiqués par Guy et al (1993) pour les 3 troupeaux avec des écarts types à peine diminués. Les poids des foies gras sont eux aussi voisins, ceux enregistrés par Guy et al (1993) étant $793 \pm 111,702 \pm 128$ et $560 \pm 101$ respectivement pour l'oie, le canard mulard et le canard de Barbarie.

La qualité technologique mesurée par les taux de fonte apparaît un peu faible pour

Tableau I. Caractéristiques ${ }^{1}$ des animaux retenus pour les analyses de composition.

\begin{tabular}{llccc} 
Espèce & $\begin{array}{c}\text { Poids avant } \\
\text { gavage }(g)\end{array}$ & $\begin{array}{c}\text { Poids en fin } \\
\text { de gavage }(g)\end{array}$ & $\begin{array}{c}\text { Poids des foies } \\
(g)\end{array}$ & $\begin{array}{c}\text { Fonte lipidique } \\
(\%)\end{array}$ \\
\hline Oie & & & & \\
Canard mulard & $5028^{\mathrm{a}} \pm 497$ & $7434^{\mathrm{a}} \pm 638$ & $768^{\mathrm{a}} \pm 143$ & $13,9^{\mathrm{a}} \pm 8,8$ \\
Canard de Barbarie & $4200^{\mathrm{b}} \pm 356$ & $6473^{\mathrm{b}} \pm 461$ & $677^{\mathrm{b}} \pm 123$ & $40,7^{\mathrm{b}} \pm 11,4$ \\
& $457^{\mathrm{b}} \pm 339$ & $6565^{\mathrm{b}} \pm 524$ & $553^{\mathrm{c}} \pm 55$ & $56,0^{\mathrm{c}} \pm 3,6$
\end{tabular}

\footnotetext{
1 Valeurs moyennes \pm écarts types $(n=9)$; tous les animaux retenus pour cette étude sont de sexe mâle. 2 Les valeurs qui sont suivies de la même lettre ne sont pas significativement différentes $(p<0,001)$.
} 
les oies sélectionnées (14\%) lorsqu'on les compare au troupeau entier (21\% : voir Guy et al, 1994), tandis qu'il n'y a aucune différence entre nos échantillonnages et les troupeaux dans le cas des canards.

Dans l'ensemble les performances notées au tableau I pour notre sélection correspondent à celles enregistrées pour les 3 troupeaux. Les oies produisent toujours les foies les plus lourds et les moins fondants. Les canards mulards et de Barbarie, de poids vifs comparables surtout en fin de gavage, divergent par leur rendement en foie gras nettement plus élevé pour les premiers que pour les seconds.

\section{Les principaux constituants des 3 types de foie gras (tableau II)}

Les foies maigres renferment très peu de lipides, essentiellement des phospholipides (Leclercq et al, 1968 ; Babilé, 1989 ; Auvergne, 1992). Globalement, les modifications engendrées par le gavage sont semblables pour les 3 types de foie gras: accroissement de la masse lipidique aux dépens des protéines et de l'eau, et faible teneur en minéraux (cendres). Cependant, les foies d'oies et de canards diffèrent significativement par leur composition. D'une manière générale le foie d'oie contient moins de lipides, laissant plus de place à l'eau, aux protéines et aux minéraux. Les foies des canards mulard et de Barbarie ne diffèrent pas entre eux.

\section{Composition des lipides hépatiques (tableau III)}

Là encore les ressemblances frappent plus que les différences. Dans les 3 types de foies les triglycérides constituent la part dominante (92 à 96\%). Cependant l'examen de la répartition des classes de lipides (tableau II) fait apparaître des différences significatives entre les 3 lots. Les lipides des foies gras des 2 types de canards contiennent plus de triglycérides ( 2 à $4 \%$ de plus), mais moins de phospholipides et moins de cholestérol.

L'écart avec l'oie est particulièrement important pour le Barbarie : teneurs réduites de plus de la moitié pour les phospholipides $(1,53$ vs $3,46 \%)$, pour le cholestérol estérifié $(0,5$ vs $1,3 \%)$ et le cholestérol total $(1,7$ vs $3,2 \%)$; toutes ces différences étant très significatives $(P<0,001)$. D'une manière générale le foie du canard mulard a une composition intermédiaire, plus proche cependant de celle du foie de Barbarie que de celle du foie d'oie. Les différences entre les 2 types de canards ne sont significatives que pour le cholestérol libre $(2 \%$ chez le mulard vs 1,2 chez le Barbarie) et le cho-

Tableau II. Composition (\%) ${ }^{1}$ des 3 types de foie.

\begin{tabular}{|c|c|c|c|c|c|}
\hline Espèces & $\underset{\star *}{\text { Lipides }}$ & $\underset{* \star}{E a u}$ & $\begin{array}{c}\text { Protéines } \\
\star\end{array}$ & $\underset{*}{C e n d r e s}$ & $\begin{array}{c}\text { Autres } \\
\text { constituants }\end{array}$ \\
\hline Oie & $54,6^{\text {a } 2} \pm 4,3$ & $32,7^{a} \pm 3,1$ & $8,3^{a} \pm 0,9$ & $0,7^{a} \pm 0,1$ & 3,7 \\
\hline Canard mulard & $60,5^{b} \pm 4,4$ & $28,5^{b} \pm 3,4$ & $6,9 b \pm 1$ & $0,6^{b} \pm 0,1$ & 3,5 \\
\hline Canard de Barbarie & $62,6^{b} \pm 1,8$ & $27,4^{b} \pm 1,8$ & $6,4^{b} \pm 0,6$ & $0,5^{b} \pm 0,1$ & 3,1 \\
\hline
\end{tabular}

\footnotetext{
1 Valeurs moyennes \pm écarts types $(n=9),{ }^{2}$ Les valeurs qui sont suivies de la même lettre ne sont pas
} significativement diffèrentes ( $\left.{ }^{*} p<0,01 ;{ }^{* *} p<0,001\right) .{ }^{3}$ Obtenus par différence, de nature indéterminée. 
Tableau III. Composition (\%) 1 des lipides hépatiques.

\begin{tabular}{lccccccc}
\hline & \multicolumn{2}{c}{$\begin{array}{c}\text { Triglycérides Phospholipides Acides gras } \\
\text { libres }\end{array}$} & $\begin{array}{c}\text { Cholestérol } \\
\text { libre }\end{array}$ & $\begin{array}{c}\text { Cholestérol } \\
\text { estérifie }\end{array}$ & $\begin{array}{c}\text { Cholestérol } \\
\text { total }^{* \star}\end{array}$ \\
\hline & & & & & & & \\
Oie & $92,2^{\mathrm{a}} \pm 1,4$ & $3,5^{\mathrm{a}} \pm 1,2$ & $1,0 \pm 0,5$ & $1,8^{\mathrm{ab}} \pm 0,4$ & $1,3^{\mathrm{a}} \pm 0,5$ & $3,2^{\mathrm{a}} \pm 0,6$ \\
Canard Mulard & $94,4^{\mathrm{b}} \pm 1,9$ & $1,95^{\mathrm{b}} \pm 0,8$ & $0,8 \pm 0,6$ & $2,0^{\mathrm{a}} \pm 0,8$ & $0,7^{\mathrm{b}} \pm 0,2$ & $2,7^{\mathrm{a}} \pm 0,8$ \\
Canard de Barbarie & $95,7^{\mathrm{b}} \pm 1,6$ & $1,5^{\mathrm{b}} \pm 0,8$ & $1,1 \pm 0,6$ & $1,2^{\mathrm{b}} \pm 0,6$ & $0,5^{\mathrm{b}} \pm 0,2$ & $1,7^{\mathrm{b}} \pm 0,6$
\end{tabular}

1 Valeurs moyennes \pm écarts types $(n=9) .2$ Les valeurs suivies de la même lettre ne sont pas significativement différentes $\left(" p<0,05 ;{ }^{* *} p<0,001\right)$.

lestérol total $(2,7 \%$ chez le mulard vs 1,7 chez le Barbarie).

\section{Composition en acides gras}

Dans le tableau IV nous comparons la composition en acides gras des lipides totaux du foie et des graisses de réserve des animaux gavés. Les graisses de réserve n'ont pas été beaucoup modifiées par le gavage : comme toujours chez les oiseaux, la proportion d'acides insaturés est élevée, l'ensemble des acides oléique (18:1) plus lino- léique (18:2) totalisant 58 à $64 \%$ des acides gras ; de ce fait le rapport acides gras insaturés/saturés est élevé, variant de 1,7 à 2,1.

D'une manière générale les lipides hépatiques (tableau IV) renferment davantage d'acides gras saturés que les graisses de réserve, la proportion d'acide stéarique (18:0) étant plus élevée $(+24$ à $+75 \%)$, tandis que la teneur en acide linoléique est considérablement réduite. Le foie d'oie diffère significativement des foies de canard, il renferme moins d'acides gras saturés (14:0, 16:0 et 18:0) et, en dépit de sa teneur plus faible en acide linoléique $(0,8$ vs $1,2 \%)$,

Tableau IV. Composition en acide gras (\% des acides gras totaux). Comparaison des foies et des tissus adipeux des oies et des canards.

\begin{tabular}{|c|c|c|c|c|c|c|c|}
\hline Acides gras & $=1 \quad 14: 0$ & $16: 0$ & $16: 1$ & $18: 0$ & $18: 1$ & $18: 2$ & $1 / S^{2}$ \\
\hline \multicolumn{8}{|c|}{ Tissu adipeux 1} \\
\hline Oie & 0,7 & 23,6 & 3,9 & 7,6 & 56,1 & 7,7 & 2,1 \\
\hline Mulard & 0,8 & 26,6 & 4,8 & 7,5 & 50,2 & 9,6 & 1,9 \\
\hline Barbarie & 1,0 & 27,6 & 4,3 & 8,8 & 46,8 & 11,5 & 1,7 \\
\hline \multicolumn{8}{|l|}{ Foie $^{3}$} \\
\hline Oie & $0,8^{a * *} \pm 0,1$ & $25,0^{a * *} \pm 2,3$ & $3,4 \pm 0,5$ & $11,6^{a b *} \pm 0,7$ & $58,5^{a^{\star *}} \pm 2,6$ & $0,8^{a^{*} \pm 0,1}$ & $1,7^{a^{*}} \pm 0,5$ \\
\hline Mulard & $0,9^{b} \pm 0,1$ & $28,8^{b} \pm 3,3$ & $4,2 \pm 1,6$ & $13,1^{b} \pm 2,6$ & $51,7^{b} \pm 3,4$ & $1,2^{b} \pm 0,3$ & $1,3^{b} \pm 0,1$ \\
\hline Barbarie & $1,2^{c} \pm 0,1$ & $31,3^{b} \pm 1,4$ & $4,2 \pm 0,4$ & $10,9^{a} \pm 0,2$ & $51,1^{\mathrm{b}} \pm 1,8$ & $1,2^{b} \pm 0,2$ & $1,3^{b} \pm 0,1$ \\
\hline
\end{tabular}

1 Les échantillons de tissus adipeux ont été mélangès pour former un pool par type de palmipède ; les foies gras ont été analysés individuellement. ${ }^{2}$ I/S est le rapport : \% d'acides gras insaturés /\% d'acides saturés. ${ }^{3}$ Les valeurs suivies de la même lettre ne sont pas significativement différentes $(* P<0,01 ; * * P<0,001$ ). 
contient plus d'insaturés grâce à l'acide oléique $(58,5 \%)$. Le profil des acides gras est identique chez le canard mulard et chez le canard de Barbarie ; la proportion d'acides saturés est augmentée ; le rapport insaturés/saturés atteint une valeur basse de 1,3 qui caractérise les graisses saturées (dans le cas du suif par exemple ce ratio est de 1,05 et n'est donc que légèrement inférieur).

\section{DISCUSSION}

Quelle que soit leur origine, les foies gras ont des caractéristiques communes. Leur teneur élevée en lipides (55 à 65\%) est due à la présence de triglycérides (plus de $90 \%$ des lipides) synthétisés à partir des glucides du régime de gavage, autrement dit à partir de l'amidon du maïs. Ceci explique l'accroissement des teneurs en acides gras saturés et en acides oléiques mono-insaturés que tous les oiseaux synthétisent aisément. Dans une certaine mesure l'acide oléique prend la place de l'acide linoléique qui, lui, fait défaut.

La teneur extrêmement faible des foies en acide linoléique vérifie toutes les études antérieures (Leclercq et al, 1968 ; Baudonnet-Lenfant, 1991). Elle n'en est pas moins suprenante, compte tenu de l'apport alimentaire assez élevé : $2 \%$ en poids du maīs sec, soit $5 \%$ environ de l'apport énergétique métabolisable, ce qui laisse supposer un transport préférentiel de l'acide linoléique vers la périphérie.

Les triglycérides qui s'accumulent dans le foie diluent les autres constituants, de sorte que la composition de l'organe sans les lipides est quasiment identique pour les canards et les oies : 66,5 à $69,5 \%$ d'eau, 16 à $19 \%$ de protéines, 1,3 à $1,4 \%$ de minéraux. De même la teneur en eau diminue lorsque la concentration en lipides augmente suivant une droite de régression qui est identique pour les trois types de foie (fig 1).

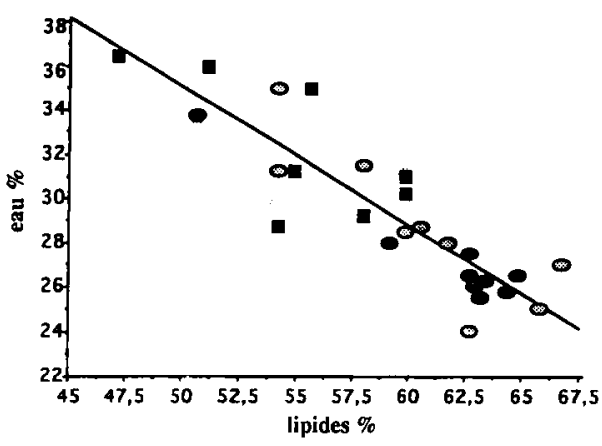

Fig 1. Corrélation lipides-eau dans les 3 espèces. Die; $\bigcirc$ mulard; $\infty$ Barbarie.

Malgré ces similitudes, les 3 types de foie ont des qualités technologiques bien différentes. Les mesures effectuées à Artiguères (fig 2) montrent que la fonte s'élève beaucoup plus vite avec le poids des foies chez le canard mulard que chez l'oie. En fait il y a 3 droites de régression différentes selon le type de foie. La rétention la plus forte est observée chez l'oie (fonte $=0,04 \mathrm{x}$ poids du foie $-9,0 ; r=0,69$ ). Chez les mulards, la fonte est plus importante dans les gros foies et la pente de la droite de régression est plus élevée (fonte $=0,07 \mathrm{x}$ poids du foie $-3,4 ; r=0,74)$. Chez le canard de Barbarie, la fonte atteint des

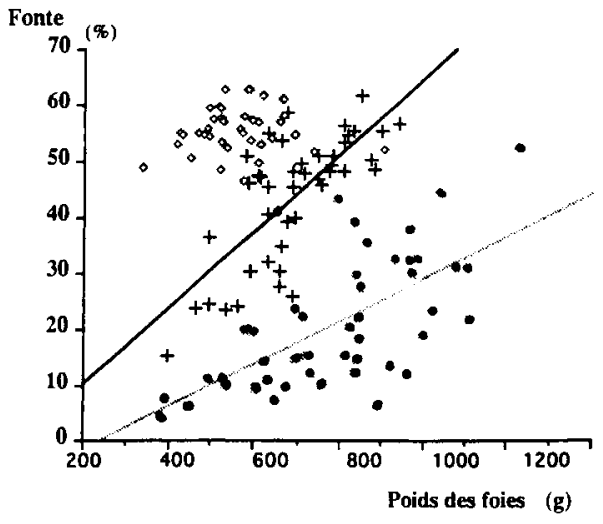

Fig 2. Relation entre la fonte lipidique et le poids du foie. $\diamond$ Barbarie; + mulard; 0 Oie. 
valeurs maximum qui ne dépendent plus du poids du foie (fonte $=0,004 \times$ poids $\mathrm{du}$ foie $+53,2 ; r=0,1$ ).

Ces différences traduisent un déséquilibre variable entre les concentrations des lipides et les autres constituants. Chez l'oie nous confirmons largement les observations antérieures montrant que le dépôt des triglycérides est associé à un accroissement important des autres constituants (Blum et al, 1990). Connaissant le poids et la composition du foie avant gavage $(70 \%$ d'eau, $20 \%$ de protéines, $4 \%$ de lipides essentiellement des phospholipides, $1,5 \%$ de cendres..., pour un poids de foie maigre de 80 à $100 \mathrm{~g}$ ), on peut estimer que le foie gras entier contient au total 3 fois plus de protéines et 4 à 5 fois plus de phospholipides que le foie maigre. Chez les canards, ces constituants s'accroissent également mais dans une moindre mesure, de sorte que les teneurs en protéines, phospholipides et cholestérol sont significativement inférieures.

En conclusion, le foie gras, quelle que soit son origine, est très riche en graisse relativement saturée. Cela est particulièrement vrai pour le foie de canard qui renferme cependant un peu moins de cholestérol, surtout le foie de Barbarie comparé au foie d'oie.

L'efficacité alimentaire (indices de consommation pour le gain de poids de gavage : 4,2 à 4,8) et l'aptitude à former un foie gras n'apparaissent pas très différentes d'une espèce à l'autre. Dans tous les cas (différences non significatives), 25 à $30 \%$ des gains de poids de gavage sont expliqués par le développement du foie gras. Un calcul simple montre que les graisses du foie représentent $8,7 \%$ de l'énergie métabolisable ingérée chez l'oie, $9,5 \%$ chez le mulard et $10,4 \%$ chez le Barbarie. Pourtant dans cette dernière espèce le poids du foie est significativement plus petit, en valeur absolue comme en pourcentage du poids vif $(8,6 \%$ vs 10,7 et $10,4 \%$ respectivement chez le mulard et l'oie). Canard maigre, plus difficile à gaver que le mulard, le Barbarie conserve plutôt mieux que les autres palmipèdes les graisses néoformées dans le foie. Mais faute d'un accroissement notable des autres constituants, les structures hépatiques assurant la cohésion des parenchymes sont insuffisantes pour retenir les lipides au cours de la cuisson. Pour éviter la fonte, il faudrait certainement se contenter d'un poids de foie réduit pour le Barbarie et seulement un peu plus élevé pour le mulard. En attendant bien entendu que la recherche fasse mieux (génétique, nutrition...) en réussissant à additionner tous les avantages chez un même animal.

\section{RÉFÉRENCES}

Auvergne A (1992) Facteurs de variation de la composition corporelle et tissulaire des canards avant et après gavage. Thèse Doct d'État, INP Toulouse, $252 \mathrm{p}$

Babilé R (1989) La production de foie gras de canards de Barbarie : aspects génétiques, nutritionnels et technologiques. Thèse Doct d'État, INP Toulouse, $302 \mathrm{p}$

Baudonnet-Lenfant C, Auvergne A, Babilé R (1991) Influence de la durée du jeûne avant l'abattage et du poids avant la mise en gavage des canards de Barbarie sur la composition chimique hépatique. Ann Zootech 40, 161-170

Blum JC, Leclercq B (1973) Nouvelles précisions sur les modifications biochimiques et histologiques du fole provoquées par le gavage. In: Journées Avicoles de Varese, Italie, 31 mai-4 juin, G Avic int 5, 2, 193-207

Blum JC, Labie C, Raynaud P (1990) Influence du poids et de la composition chimique du foie gras d'oie sur la fonte mesurée après stérilisation. Sci Aliments 10, 543-554

Folch J, Lees M, Sloane-Stanley C (1957) A simple method for the isolation and purification of total lipids from animal tissues. $J \mathrm{Biol}$ Chem 226, 497-509

Guy G, Rousselot-Pailley D, Gourichon D (1994) Comparaison des performances quantitatives et qualitatives du gavage de l'oie, du canard mulard et du canard de Barbarie. Ann Zootech (sous presse) 
Hermier D, Saadoun A, Salichon MR, Sellier N Rousselot-Pailley D, Chapman MJ (1991) Plasma lipoproteins and liver lipids in two breeds of geese with different susceptibility to hepatic steatosis: changes induced by development and force-feeding. Lipids 26(5), 331339

Leclercq B, Durand G, Delpech P, Blum JC (1968) Note préliminaire sur l'évolution des consti- tuants biochimiques du foie au cours du gavage de l'oie. Ann Biol Anim Bioch Biophys 8(4), 549-556

Nir I, Nitsan Z (1976) Goose fatty liver composition. Ann Zootech 25, 461-470

Rémignon H (1990) Évolution au cours du gavage de la composition corporelle et hépatique du canard de Barbarie. Mémoire DEA, INP Toulouse, $46 \mathrm{p}$ 